\title{
Study of the Nonlinear Dropping Shock Response of Expanded Foam Packaging System
}

\author{
Huan-xin Jiang, ${ }^{1}$ Yong $\mathrm{Zhu},{ }^{2}$ and Li-xin Lu ${ }^{1}$ \\ ${ }^{1}$ School of Mechanical Engineering, Jiangnan University, Wuxi 214122, China \\ ${ }^{2}$ Department of Packaging Engineering, Jinan University, Zhuhai 519070, China \\ Correspondence should be addressed to Yong Zhu; zymmpi@126.com and Li-xin Lu; lulx@jiangnan.edu.cn
}

Received 16 May 2013; Revised 19 July 2013; Accepted 21 July 2013

Academic Editor: Jun Wang

Copyright (c) 2013 Huan-xin Jiang et al. This is an open access article distributed under the Creative Commons Attribution License, which permits unrestricted use, distribution, and reproduction in any medium, provided the original work is properly cited.

\begin{abstract}
The variational iteration method-2 (VIM-2) is applied to obtain approximate analytical solutions of EPS foam cushioning packaging system. The first-order frequency solution of the equation of motion was obtained and compared with the numerical simulation solution solved by the Runge-Kutta algorithm. The results showed the high accuracy of this VIM with convenient calculation.
\end{abstract}

\section{Introduction}

Dropping is an unavoidable situation for a packaged product while delivered, which is investigated by many researchers [1-3]. In most cases, the constitutive model of cushioning package materials is strong nonlinear.

The variational iteration method (VIM), first proposed by Professor He [4], can be used to solve some strong nonlinear engineering problems. VIM can avoid some defects of Adomian method and some other kinds of perturbation methods. And by a few steps of iteration, the convergence solution can be easily obtained. After investigated in some VIM researching, He and $\mathrm{Wu}$ [5] developed this method into a general basic framework. Khan et al. [6] researched the application of VIM in fractional nonlinear differential equations with initial boundary problem. Rezazadeh et al. [7] studied the parametric oscillation of an electrostatically actuated microbeam using variational iteration method. Bildik et al. $[8,9]$ compared the VIM, differential transform method, and the Adomian decomposition method for partial nonlinear differential equations, and the results showed that VIM was more reliable. And in the packaging dynamics area, Wang et al. [10] obtained the inner-resonance conditions of tangent cushioning packaging system by applying VIM with good agreement. Jafari and Khalique [11] applied the variational iteration methods for solving fuzzy differential equations. Most recently, $\mathrm{Wu}$ soluted the fractional heat equations by variational iteration method [12].
According to [4], if a differential equation can be written as

$$
\ddot{u}+f(u, \dot{u}, \ddot{u})=0,
$$

the corresponding iteration equation can be identified as

$$
u_{n+1}(t)=u_{n}(t)+\int_{0}^{t}(s-t)\left\{\ddot{u}_{n}(s)+f\left(u_{n}, \dot{u}_{n}, \ddot{u}_{n}\right)\right\} \mathrm{d} s .
$$

This presented paper investigated for the first time the applicability and the validity of this VIM-2 for EPS foam cushioning packaging system. Besides, in order to show the accuracy of this method, some specific parameters were used in the constitutive equation based on real situation, and solutions of VIM-2 and Runge-Kutta method were compared.

\section{EPS Foam Nonlinear Packaging System}

While dropping, the nondimensional motive equation of EPS foam packaging system can be described as [13]:

$$
\ddot{X}+\tanh X+\lambda_{2} \tan \left(\lambda_{1} X\right)+\lambda_{3} \tan ^{3}\left(\lambda_{1} X\right)=0,
$$

with initial boundary conditions:

$$
X(0)=0, \quad \dot{X}(0)=V,
$$

where $X$ is the nondimensional displacement while dropping, $V$ is the nondimensional initial velocity, and $\lambda_{1}, \lambda_{2}$, and $\lambda_{3}$ are the nondimensional system parameters. 
By the fifth-order Taylor series, (5) can be expanded as the following to simplify the calculation:

$$
\ddot{X}+\kappa_{1} X+\kappa_{2} X^{3}+\kappa_{3} X^{5}=0 .
$$

In order to simplify the calculation, we set

$$
\begin{gathered}
\kappa_{1}=\left(\lambda_{1} \lambda_{2}+1\right), \\
\kappa_{2}=\left(\frac{\lambda_{1}^{3} \lambda_{2}}{3}+\lambda_{1}^{3} \lambda_{3}-\frac{1}{3}\right), \\
\kappa_{3}=\left(\frac{2 \lambda_{1}^{5} \lambda_{2}}{15}+\lambda_{1}^{5} \lambda_{3}+\frac{2}{15}\right) .
\end{gathered}
$$

Thus, with the initial solution $X_{0}=A \sin \Omega t$, (2) can be rewritten and solved as

$$
\begin{aligned}
X_{1}(t)= & X_{0}(t)+\int_{0}^{t}(s-t)\left\{\ddot{X}_{0}+\kappa_{1} X_{0}+\kappa_{2} X_{0}^{3}+\kappa_{3} X_{0}^{5}\right\} \\
= & X_{0}(t)+\int_{0}^{t}(s-t) \\
& \times\left\{\left[\frac{5 \kappa_{3}}{8} A^{5}+\frac{3 \kappa_{2}}{4} A^{3}+\left(\kappa_{1}-\Omega^{2}\right) A\right] \sin \Omega s\right. \\
& +\left(-\frac{5 \kappa_{3}}{16} A^{5}-\frac{\kappa_{2}}{4} A^{3}\right) \sin 3 \Omega s \\
& \left.+\frac{\kappa_{3}}{16} A^{5} \sin 5 \Omega s\right\} \mathrm{d} s . \\
& \left(\frac{5 \kappa_{3}}{8} A^{5}+\frac{3 \kappa_{2}}{4} A^{3}+\kappa_{1} A\right) \sin \Omega t \\
& +\left(-\frac{5 \kappa_{3}}{144} A^{5}-\frac{\kappa_{2}}{36} A^{3}\right) \sin 3 \Omega t+\frac{\kappa_{3}}{400} A^{5} \sin 5 \Omega t \\
& +\frac{15 \Omega^{2}-8 \kappa_{3} A^{4}-10 \kappa_{2} A^{2}-15 \kappa_{1}}{15 \Omega} A t .
\end{aligned}
$$

In order to eliminate the secular term, the coefficient of $t$ must be zero. Thus,

$$
15 \Omega^{2}-8 \kappa_{3} A^{4}-10 \kappa_{2} A^{2}-15 \kappa_{1}=0,
$$

which can be solved to obtain the frequency $\Omega$.

\section{Results}

In order to verify the previous method, the approximate solution by the new VIM was compared with the numerical solution solved by the Runge-Kutta method, as illustrated in Table 1, and the results show that for different parameters, the VIM solutions are all in good agreement with the numerical solutions which can be almost equal to the exact solution.

\section{Conclusions}

The dropping shock equation of polymer-based packaging system was soluted by the VIM-2. The first-order frequency
TABLE 1: Comparison of the VIM method with the numerical method by Runge-Kutta method.

\begin{tabular}{llll}
\hline Parameters & $\Omega_{\mathrm{VIM}}$ & $\Omega_{\text {num }}$ & Error, \% \\
\hline$\lambda_{1}=0.5$ & & & \\
$\lambda_{2}=2, \lambda_{3}=5$ & 1.4434 & 1.4742 & 2.089268756 \\
$\lambda_{2}=2, \lambda_{3}=10$ & 1.4995 & 1.5458 & 2.995212835 \\
$\lambda_{2}=5, \lambda_{3}=5$ & 1.8921 & 1.9037 & 0.609339707 \\
$\lambda_{2}=5, \lambda_{3}=20$ & 1.9699 & 2.0103 & 2.009650301 \\
\hline$\lambda_{1}=1$ & & & \\
$\lambda_{2}=2, \lambda_{3}=5$ & 1.9350 & 2.0761 & 6.796397091 \\
$\lambda_{2}=2, \lambda_{3}=10$ & 2.0741 & 2.2712 & 8.678231772 \\
$\lambda_{2}=5, \lambda_{3}=5$ & 2.5634 & 2.6110 & 1.8230563 \\
$\lambda_{2}=5, \lambda_{3}=20$ & 2.7688 & 2.8965 & 4.408769204 \\
\hline$\lambda_{1}=2$ & & & \\
$\lambda_{2}=2, \lambda_{3}=5$ & 2.7222 & 3.2654 & 16.63502174 \\
$\lambda_{2}=2, \lambda_{3}=10$ & 3.0180 & 3.6363 & 17.00354756 \\
$\lambda_{2}=5, \lambda_{3}=5$ & 3.6235 & 3.8534 & 5.966159755 \\
$\lambda_{2}=5, \lambda_{3}=20$ & 4.0809 & 4.5069 & 9.452173334 \\
\hline
\end{tabular}

solution of the equation of motion was obtained and compared with the numerical simulation solution solved by the Runge-Kutta algorithm. The results showed the high accuracy of this VIM-2 with convenient calculation.

\section{References}

[1] R. D. Mindlin, "Dynamics of package cushioning," Bell System Technical Journal, vol. 24, article 3, pp. 353-461, 1945.

[2] Z.-W. Wang, "On evaluation of product dropping damage," Packaging Technology and Science, vol. 15, no. 3, pp. 115-120, 2002.

[3] J. Wang, F. Duan, J. H. Jiang, and L. X. Lu, "Dropping damage evaluation for a hyperbolic tangent nonlinear system with a critical component," Journal of Vibration and Control, vol. 18, pp. 1417-1421, 2012.

[4] J.-H. He, "Variational iteration method-a kind of non-linear analytical technique: some examples," International Journal of Non-Linear Mechanics, vol. 34, no. 4, pp. 699-708, 1999.

[5] J.-H. He and X.-H. Wu, "Variational iteration method: new development and applications," Computers \& Mathematics with Applications, vol. 54, no. 7-8, pp. 881-894, 2007.

[6] Y. Khan, N. Faraz, A. Yildirim, and Q. Wu, "Fractional variational iteration method for fractional initial-boundary value problems arising in the application of nonlinear science," Computers \& Mathematics with Applications, vol. 62, no. 5, pp. 2273-2278, 2011.

[7] G. Rezazadeh, H. Madinei, and R. Shabani, "Study of parametric oscillation of an electrostatically actuated microbeam using variational iteration method," Applied Mathematical Modelling, vol. 36, no. 1, pp. 430-443, 2012.

[8] N. Bildik and A. Konuralp, "The use of variational iteration method, differential transform method and adomian decomposition method for solving different types of nonlinear partial differential equations," International Journal of Nonlinear Sciences and Numerical Simulation, vol. 7, no. 1, pp. 65-70, 2006.

[9] N. Bildik, A. Konuralp, and S. Yalçınbaş, "Comparison of Legendre polynomial approximation and variational iteration method 
for the solutions of general linear Fredholm integro-differential equations," Computers \& Mathematics with Applications, vol. 59, no. 6, pp. 1909-1917, 2010.

[10] J. Wang, Y. Khan, R.-H. Yang, L.-X. Lu, Z.-W. Wang, and N. Faraz, "A mathematical modelling of inner-resonance of tangent nonlinear cushioning packaging system with critical components," Mathematical and Computer Modelling, vol. 54, no. 11-12, pp. 2573-2576, 2011.

[11] H. Jafari and C. Khalique, "Homotopy perturbation and variational iteration methods for solving fuzzy differential equations," Communications in Fractional Calculus, vol. 3, no. 1, pp. 38-48, 2012.

[12] G. C. Wu, "Laplace transform overcoming principal drawbacks in application of the variational iteration method to fractional heat equations," Thermal Science, vol. 6, no. 4, pp. 1257-1261, 2012.

[13] J. Wang, L. Lu, H. Jiang, and Y. Zhu, "Nonlinear response of strong nonlinear system arisen in polymer cushion," Abstract and Applied Analysis, vol. 2013, Article ID 891914, 3 pages, 2013. 


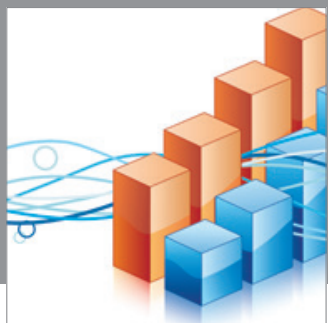

Advances in

Operations Research

mansans

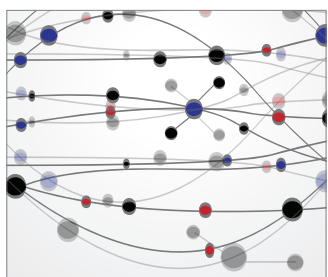

The Scientific World Journal
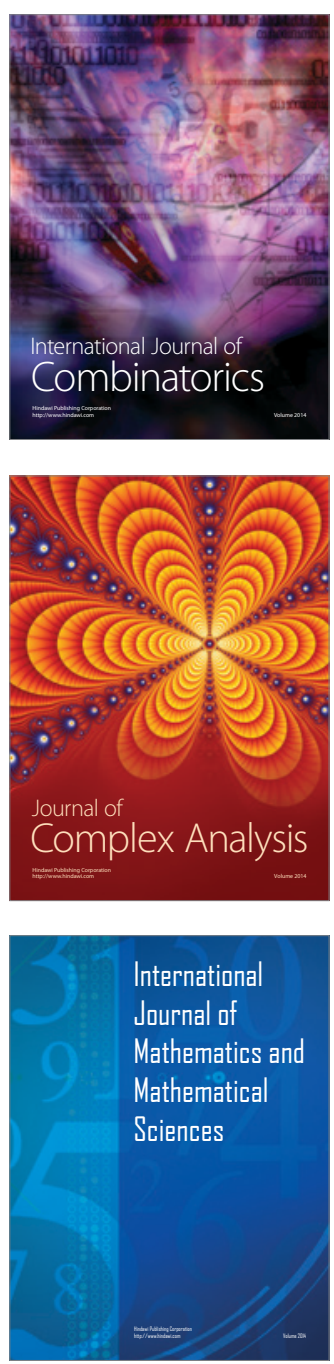
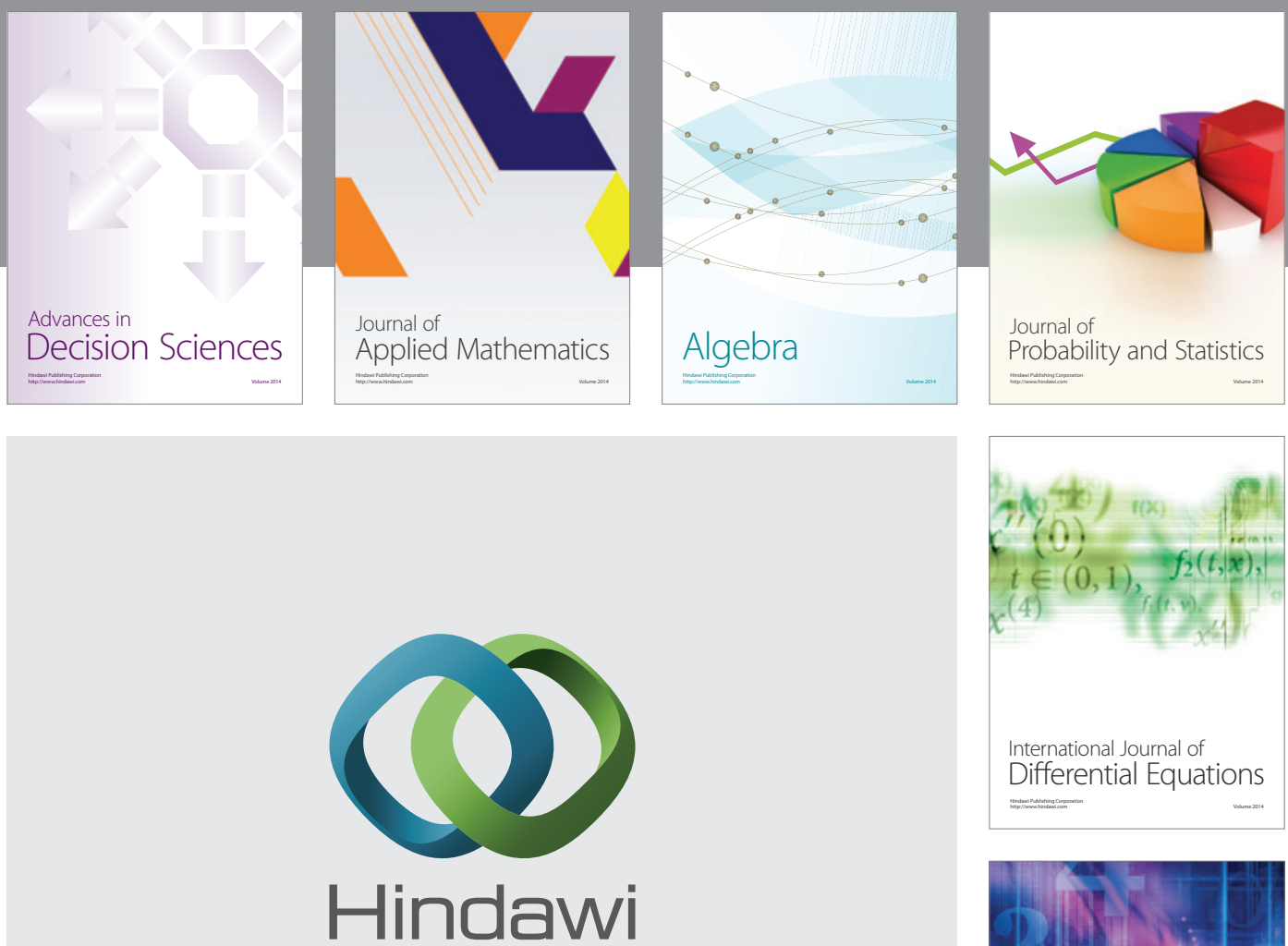

Submit your manuscripts at http://www.hindawi.com
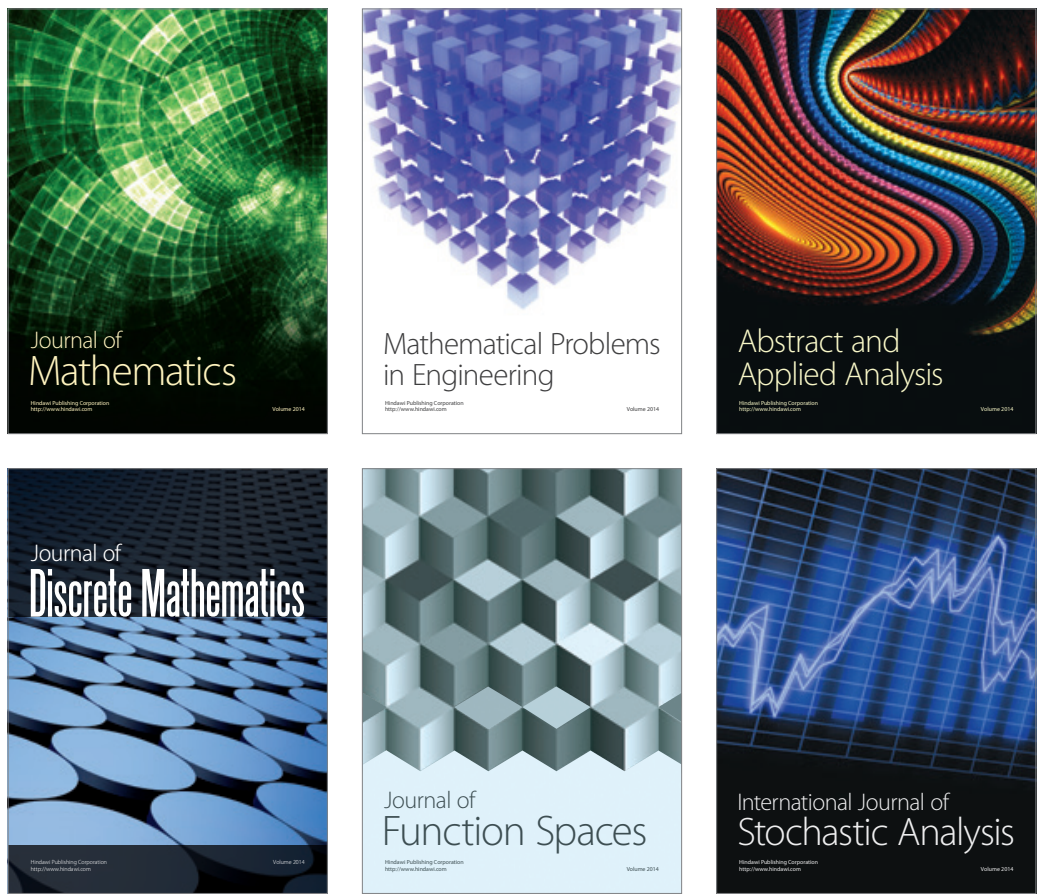

Journal of

Function Spaces

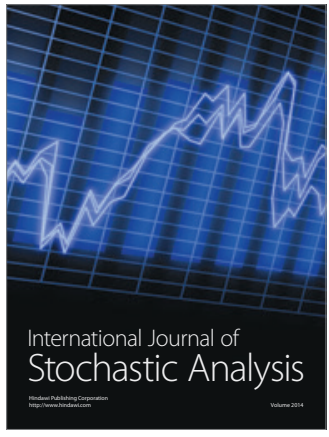

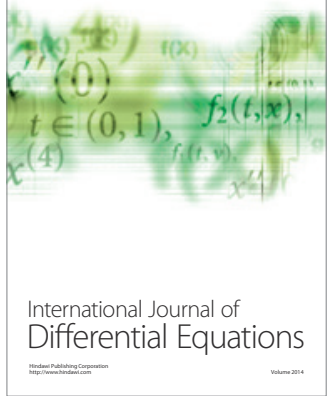
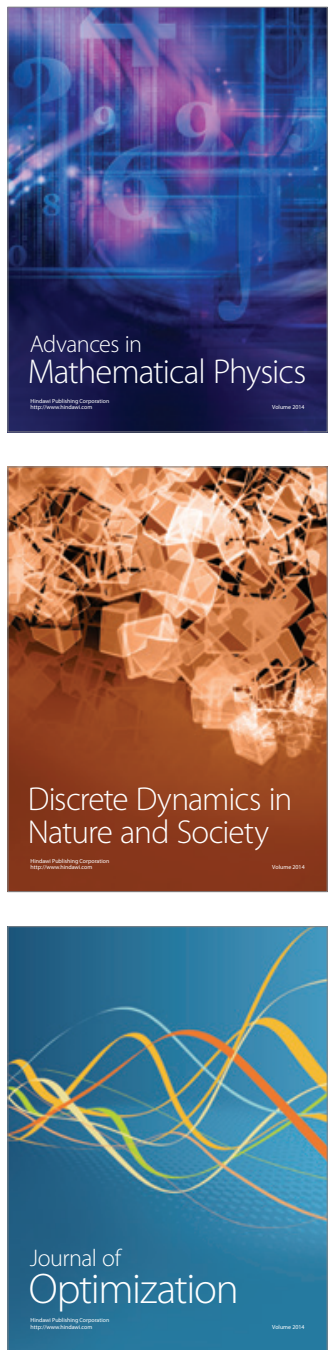